\title{
Reduction Theory for Fuchsian Groups
}

\author{
Svetlana Katok
}

Department of Mathematics, University of California, Los Angeles, CA 90024, USA

\section{Introduction}

Let $\Gamma$ be any Fuchsian group, i.e., a discrete subgroup of the group of isometries of the hyperbolic plane $H^{2}$. We consider the unit disc $D=\{z \in \mathbf{C},|z|<1\}$ endowed with the Poincaré metric $d s=\frac{2|d z|}{1-|z|^{2}}$ as a model of the hyperbolic plane. The geodesics for this metric are circular arcs orthogonal to $S^{1}=\partial D=\{z \in \mathrm{C},|z|=1\}$. Two geodesics can intersect at most once. The group $\Gamma$ acts on $D$ by linear fractional transformations and can be represented by matrices $\left(\begin{array}{ll}a & \bar{c} \\ c & \bar{a}\end{array}\right)$ with $a, c \in \mathbf{C}$ and $a \bar{a}-c \bar{c}=1$. The unit circle $S^{1}$ is a fixed circle for the group $\Gamma, \Gamma: S^{1} \rightarrow S^{1}$. All the necessary information about Fuchsian groups can be found in $[2]$.

In this paper we develop a so-called reduction theory for Fuchsian groups $\Gamma$ with compact quotient $\Gamma \backslash D$. We assume for convenience that 0 is not an elliptic point of $\Gamma$, i.e. $c \neq 0$, for $\left(\begin{array}{ll}a & \bar{c} \\ c & \bar{a}\end{array}\right) \in \Gamma$. Obviously, every $\Gamma$ is conjugate to a group satisfying this property. This theory serves the same purpose as Gauss reduction theory for $S L_{2}(Z)$ based on continued fractions. An important ingredient in the argument is a construction of two expanding maps on the boundary $f_{ \pm}: S^{\mathbf{1}} \rightarrow S^{1}$ associated to the group $\Gamma$. This construction is a generalization of that used by Bowen and Series in [1].

\section{Construction of the Fundamental Region $R_{0}$ and the Special Polygon $R$}

Definition. Let $\gamma=\left(\begin{array}{ll}a & b \\ c & d\end{array}\right) \in \Gamma$. The circle $J(\gamma)=\{z \in D,|c z+d|=1\}$ is called the isometric circle of $\gamma$.

Since $\gamma^{\prime}(z)=(c z+d)^{-2}, \gamma$ expands Euclidean distances within $J(\gamma)$ and contracts outside. 
Let $R_{0}$ be the intersection of $D$ with the exteriors of all isometric circles $J(\gamma)$, $\gamma \in \Gamma$. The region $R_{0}$ constitutes a fundamental region for $\Gamma$. Since $\Gamma$ is finitely generated and contains no parabolic elements, the boundary of $R_{0}$ consists of a finite number geodesic arcs with vertices inside $S^{1}$ [2, Theorem 15, 16, Sect. 34]. The images of $R_{0}$ under $\Gamma$ exactly fill up $D$. Each side $s$ of $R_{0}$ is identified with another side $s^{\prime}$ by an element $\gamma(s), s \subset J(\gamma(s))$ and $s^{\prime} \subset J\left(\gamma^{-1}(s)\right)=\gamma(s)(J(\gamma(s)))$. The set $\left\{\gamma(s), s\right.$ is a side of $\left.R_{0}\right\}$ forms a set of generators for $\Gamma$ [2, Sect. 23], and $R_{0}$ can be regarded as all that part of $D$ which is exterior to $J(\gamma(s))$, s is a side of $R_{0}$. In order to construct the fundamental region $R_{0}$, we list elements of the group $\Gamma$ as follows. Given any $A>0$ there are only finitely many elements of $\Gamma$ with $|a|<A$. This follows from the equality $|a|^{2}-|c|^{2}=1$ and the discreteness of the group $\Gamma$. (If $\Gamma$ is an arithmetic group then after a suitable conjugation we will have $|a|^{2},|c|^{2}$ in $\frac{1}{N} \mathbf{Z}$ for some integer $N$; see the examples in Sect. 5.) We can thus list elements of $\Gamma$ in increasing order of $|a|$. This list will eventually include all elements of $\Gamma$. Taking isometric circles for the elements according to their order we shall obtain the fundamental region $R_{0}$ as described above after a finite number of steps. Indeed the distance from the isometric circle $J(\gamma), \gamma=\left(\begin{array}{ll}a & \bar{c} \\ c & \bar{a}\end{array}\right)$ to the center of $D$ is equal to $(|a|-1) /|c|$ which tends to 1 as $|a| \rightarrow \infty$. Thus isometric circles with sufficiently large $|a|$ cannot contribute to the boundary of the compact fundamental region $R_{0}$. Examples of fundamental regions for some arithmetic groups are given in Sect. 5 .

For each geodesic arc $J(\gamma(s))$ we consider the smaller of two arcs of $\partial D$ having the same end points. Since all the vertices of $R_{0}$ lie inside $\partial D$ these chosen arcs form a cover of $\partial D$. We can always choose a subcover of this cover in such a way that no two non-consecutive arcs intersect by deleting some "extra" arcs.

Definition. We shall call a polygon $R \subset D$ a special polygon associated to $\Gamma$ if it satisfies the following properties:

i) $R$ has finite number of vertices and they all lie inside $D$;

ii) All sides of $R$ belong to isometric circles of some elements of $\Gamma$;

iii) Isometric circles containing any two non-consecutive sides of $R$ do not intersect.

Obviously, the polygon formed by the isometric circles corresponding to the arcs of a subcover constructed above is a special polygon.

\section{Construction of the Maps $f_{+}$and $f_{-}$}

Let $R$ be a special polygon associated to $\Gamma$. Its sides and the end points of the corresponding geodesic arcs are labeled in the anticlockwise direction by $s_{1}, \ldots, s_{n}$ and $\left[P_{1}, Q_{1}\right], \ldots,\left[P_{n}, Q_{n}\right]$ respectively. For each $\operatorname{arc}\left[P_{i}, Q_{i}\right] \subset S^{1}$ we choose an arc $\left[P_{i}^{\prime}, Q_{i}\right] \subset S^{1}$ inside $\left[P_{i}, Q_{i}\right]$ in such a way that the order of the points $P_{1}^{\prime}, Q_{n}^{\prime}, P_{2}^{\prime}, Q_{1}^{\prime}, \ldots, P_{n}^{\prime}, Q_{n-1}^{\prime}$, is the same as the order of the points $P_{1}, Q_{n}, P_{2}, Q_{1}, \ldots, P_{n}, Q_{n-1}$ (see Fig. 1), so that the arcs $\left[P_{i}^{\prime}, Q_{i}\right]$ still form a cover of $S^{1}$. 
Fig. 1

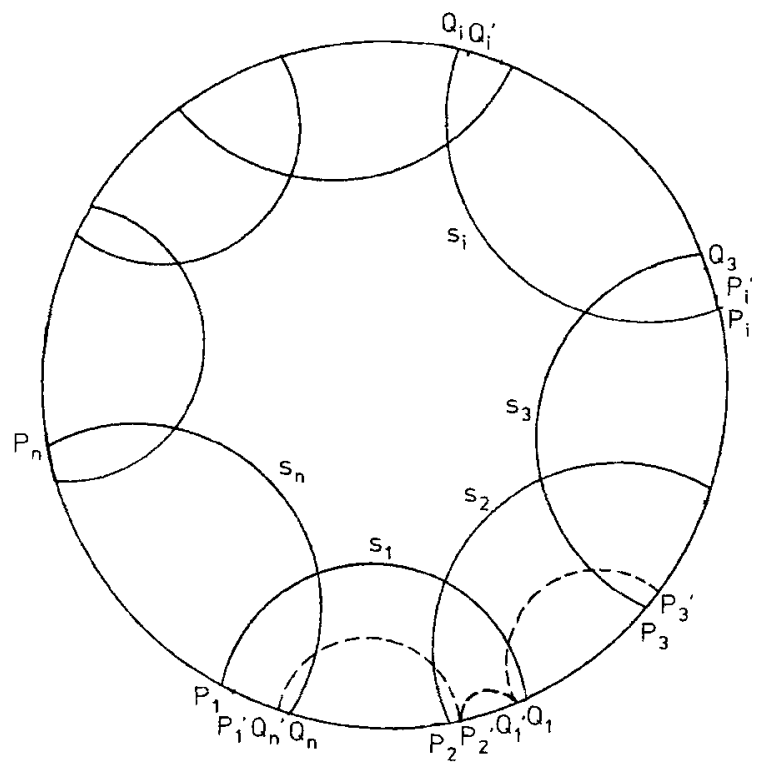

We define two partitions of $S^{\mathbf{1}}$ :

$$
M^{+}=\left\{\omega_{i}^{+}\right\}_{i=1}^{n}, \quad \omega_{i}^{+}=\left[P_{i}^{\prime}, P_{i+1}^{\prime}\right), \quad i \in \mathbf{Z} / n \mathbf{Z}
$$

and

$$
M^{-}=\left\{\omega_{j}^{-}\right\}_{j=1}^{n}, \quad \omega_{j}^{-}=\left[Q_{j-1}^{\prime}, Q_{j}^{\prime}\right), \quad j \in \mathbf{Z} / n \mathbf{Z},
$$

and two piecewise continuous maps $f_{+}, f_{-}: S^{\mathbf{1}} \rightarrow S^{\mathbf{1}}$ :

$$
\begin{array}{lll}
f_{+}(x)=\gamma_{i}(x), & \text { if } & x \in \omega_{i}^{+} ; \\
f_{-}(x)=\gamma_{j}(x), & \text { if } & x \in \omega_{j}^{-} .
\end{array}
$$

Since $\omega_{i}^{+} \subset\left[P_{i}^{\prime}, Q_{i}^{\prime}\right]$, it lies inside the isometric circle $J\left(\gamma_{i}\right)$ and we have for $x \in \omega_{i}^{+}$ $\left|f_{+}^{\prime}(x)\right|=\left|\gamma_{i}^{\prime}(x)\right|>\lambda_{i}>1$. Similarly, for $x \in \omega_{j}^{-}$we have $\left|f_{-}^{\prime}(x)\right|>\lambda_{j}>1$. Taking into account that $R$ has a finite number of sides, we have the following result.

Lemma 1. The maps $f_{+}$and $f_{-}$are expanding, i.e., there exists $\lambda>1$ such that $\left|f_{+}^{\prime}(x)\right|>\lambda$ for $x \neq P_{i}^{\prime}, i=1, \ldots, n$, and $\left|f_{-}^{\prime}(x)\right|>\lambda$ for $x \neq Q_{i}^{\prime}, i=1, \ldots, n$.

Lemma 2. Suppose $[x, y] \subset \omega_{i}^{+}$and let $c$ denote one of the symbols,+- . Then either $f_{+}(x)$ and $f_{+}(y)$ belong to different elements of $M^{c}$ or, if $f_{+}(x)$ and $f_{+}(y)$ belong to the same element $\omega_{j}^{c}$ of $M^{c}$, then $f_{+}([x, y]) \subset \omega_{j}^{c}$.

Proof. Suppose $f_{+}(x) \in \omega_{j}^{c}, f_{+}(y) \in \omega_{j}^{c}$, but $f_{+}([x, y]) \notin \omega_{j}^{c}$. For $t \in \omega_{i}^{+}, f_{+}(t)=\gamma_{i}(t)$. We have $\omega_{i}^{+} \subset\left[P_{i}, Q_{i}\right] \cdot \gamma_{i}\left(\left[P_{i}, Q_{i}\right]\right)$ is an arc of $S^{1}$ lying outside $J\left(\gamma_{i}^{-1}\right)=\gamma_{i}\left(J\left(\gamma_{i}\right)\right)$ and therefore does not cover the whole circle $S^{1}$. Since $\gamma_{i}(t)$ is continuous and monotone on the arc $\left[P_{i}, Q_{i}\right], f_{+}([x, y])$ does not cover the whoie circle $S^{1}$, and the 
Fig. 2

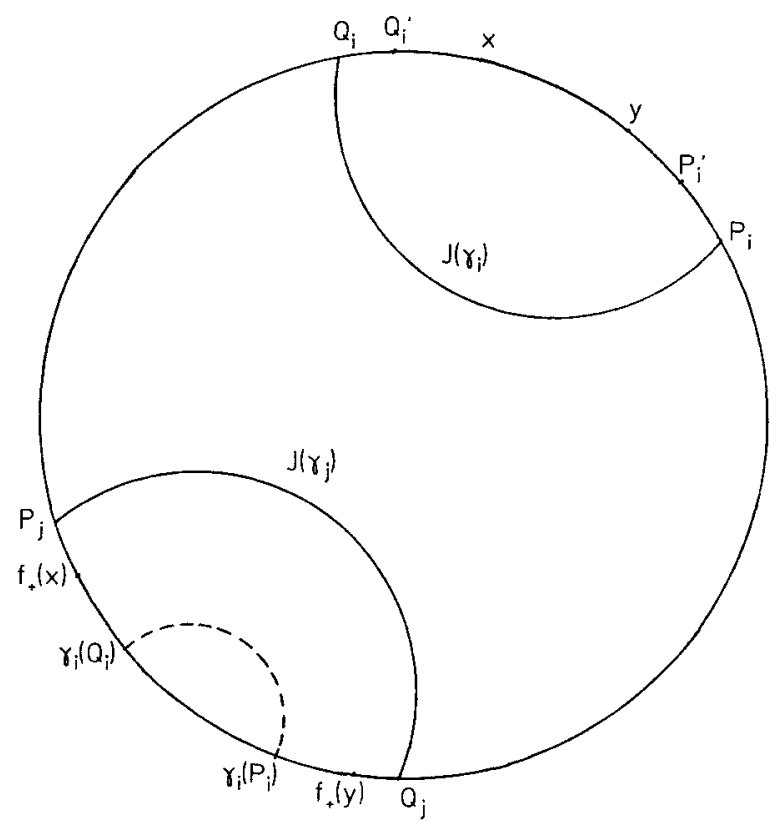

assumption forces $\gamma_{i}\left(P_{i}\right)$ and $\gamma_{i}\left(Q_{i}\right)$ to lie inside $\omega_{j}^{c}$ (see Fig. 2). Since $\omega_{j}^{+} \subset\left[\mathrm{P}_{j}, \mathrm{Q}_{j}\right]$ and $\omega_{j}^{-} \subset\left[P_{j}, Q_{j}\right]$, we have $J\left(\gamma_{i}^{-1}\right)$ lies within $J\left(\gamma_{j}\right)$, which contradicts the properties of the fundamental region $R_{0}$, described in Sect. 1.

Theorem. Let $x, y \in \omega_{i}^{+}$. There exists a finite sequence $n_{1}, n_{2}, \ldots, n_{k}$ of positive integers such that $f_{ \pm}^{n_{k}}, \ldots, f_{-}^{n_{2}} f_{+}^{n_{1}}(x)$ and $f_{ \pm}^{n_{k}}, \ldots, f_{-}^{n_{2}} f_{+}^{n_{1}}(y)$ belong to different elements of both partitions $M^{+}$and $M^{-}$.

Proof. There exists an integer $n_{1}$ such that for $i<n_{1} f_{+}^{i}([x, y]) \subset \omega_{k_{i}}^{+}$and $f_{+}^{n_{1}}(x)$ and $f_{+}^{n_{1}}(y)$ belong to different elements of the partition $M^{+}$. Otherwise, according to Lemma 2, we have $f_{+}^{n}([x, y]) \subset \omega_{k_{n}}^{+}$for all $n>0$, which contradicts the fact that $f_{+}$is expanding (Lemma 1). Let $f_{+}^{n_{1}}(x)=x_{1}, f_{+}^{n_{1}}(y)=y_{1}$. Suppose $x_{1}, y_{1} \subset \omega_{j}^{-}$. According to Lemma $2 f_{+}^{n_{1}}([x, y]) \subset \omega_{j}^{-}$, i.e. $f_{+}^{n_{1}}([x, y])=\left[x_{1}, y_{1}\right]$. Applying the same argument to the arc $\left[x_{1}, y_{1}\right]$ we find $n_{2}>0$ such that for $i<n_{2} f_{-}^{i}\left(\left[x_{1}, y_{1}\right]\right) \subset \omega_{k_{i}}^{-}$and $f_{-}^{n_{2}}\left(x_{1}\right)$ and $f_{-}^{n_{2}}\left(y_{1}\right)$ belong to different elements of the partition $M^{--}$. If $f_{-}^{n_{2}}\left(x_{1}\right)=x_{2}$ and $f_{-}^{n_{2}}\left(y_{1}\right)=y_{2}$ belong to the same element of $M^{+}$, so does the arc $f_{-}^{n_{2}}\left(\left[x_{1}, y_{1}\right]\right)$ $=\left[x_{2}, x_{2}\right]$. Since both $f_{+}$and $f_{-}$are expanding (Lemma 1), the length of $\left[x_{2}, y_{2}\right]$ is at least $\lambda$ times the length of $[x, y]$ with a fixed $\lambda>1$. This consideration shows that after a finite number of steps we obtain a pair of points $x_{k}, y_{k}$ belonging to different elements of both $M^{+}$and $M^{-}$.

\section{Reduction Theory and Coding of Geodesics}

Any hyperbolic element $\gamma \in \Gamma(|\operatorname{tr} \gamma|>2)$ has two hyperbolic fixed points on $S^{1}$, one repulsive and one attractive. We shall denote them by $w_{1}$ and $w_{2}$. Let $C(\gamma)$ be the oriented geodesic on $D$ from $w_{1}$ to $w_{2}$. It is called the axis of $\gamma$ and clearly it is 
$\langle\gamma\rangle$-invariant. We shall call a hyperbolic element $\gamma$ reduced if $C(\gamma)$ intersects the given fundamental region $R_{0}$ (see Sect. 1). $C(\gamma)$ becomes a closed geodesic in $\Gamma \backslash D$ and it can be coded according to the order it intersects the sides of $R_{0}$. This idea goes back to Morse [4] (see also [5, p. 104]). More precisely, suppose $C(\gamma)$ enters $R_{0}$ through the side $s_{i}$ and leaves it through the side $s_{j}$. The side $s_{j}$ is identified in $R_{0}$ with the side $s_{j}^{\prime}$ by the transformation $\gamma_{j}$, therefore the transformation $\gamma_{j} \gamma \gamma_{j}^{-1}=\gamma^{\prime}$ will have its axis entering $R_{0}$ through $s_{j}^{\prime}$. The first symbol in the code will be $j$, and we continue the process with $C\left(\gamma^{\prime}\right)$ instead of $C(\gamma)$. Since the geodesic is closed, the code will be periodic. If two elements $\gamma_{0}$ and $\gamma_{1}$ are conjugate in $\Gamma$, i.e., $\gamma_{1}=\gamma \gamma_{0} \gamma^{-1}$ for some $\gamma \in \Gamma$, then $C\left(\gamma_{1}\right)=\gamma C\left(\gamma_{0}\right)$. There is 1-1 correspondence between conjugacy classes of hyperbolic elements in $\Gamma$ and closed geodesics in $\Gamma \backslash D$. If two hyperbolic elements are conjugate in $\Gamma$, their closed geodesics in $R_{0}$ coincide and therefore their codes differ by a cyclic permutation. Conversely, if two closed geodesics have the same code, we can make a homotopy between them along each pair of corresponding sides. Therefore the code determines the free homotopy class of a closed geodesic, and since there is only one closed geodesic in each free homotopy class, those geodesics coincide. If two hyperbolic elements having the same trace have the same code (up to a cyclic permutation), they correspond to the same closed geodesic and therefore are conjugate in $\Gamma$. If two hyperbolic elements of different trace have the same code, then both are conjugate to powers of the same primitive hyperbolic element having the same code. We know that each hyperbolic element is conjugate in $\Gamma$ to a reduced one. The goal of a reduction theory is to give an algorithm producing this conjugation.

We shall define a compact region $D_{0}$ associated to the special polygon $R$ described in Sect. 1. Consider $2 n$ geodesics connecting each pair of the consecutive points $P_{i}^{\prime}, Q_{i-1}^{\prime}$ and $Q_{i}^{\prime}, P_{i+2}^{\prime}, i \in \mathbf{Z} / n \mathbf{Z}$ (see Fig. 1). Let $D_{0}$ be the smallest circle concentric with the principal circle $S^{\mathrm{l}}$ which intersects all those $2 n$ geodesic circles. $D_{0}$ is completely covered by $R_{0}$ and a finite number of its images under elements of $\Gamma$. We shall call a hyperbolic element $\gamma$ almost reduced if $C(\gamma)$ intersects the compact region $D_{0}$. The reduction algorithm we are about to describe assumes that we know the following data about the group $\Gamma$ :

1. a list of elements of $\Gamma$, the fundamental region $R_{0}$ as in Sect. 1 and generators of the group $\Gamma$;

2. the special polygon $R$ described in Sect. 1;

3. the compact region $D_{0}$ as above and a finite set of elements $\gamma_{(i)} \in \Gamma$, $i=1, \ldots, N$ such that $\bigcup_{i=1}^{N} \gamma_{(i)} R_{0} \supset D_{0}$.

We shall describe now the reduction algorithm, starting from a hyperbolic element $\gamma$ :

Step 1 . If the end points $w_{1}, w_{2}$ of $C(\gamma)$ (i.e., the hyperbolic fixed points of $\gamma$ ) belong to the same element $\omega_{i}^{+} \in M^{+}$, then conjugate $\gamma$ by $\gamma_{i}=f_{+}$. This replaces $\gamma$ by a new element with the fixed points $f_{+} w_{1}, f_{+} w_{2}$. If these points belong to the same element of $\mathrm{M}^{+}$, repeat Step 1; otherwise proceed to Step 2.

Step 2. If the end points $w_{1}, w_{2}$ of $C(\gamma)$ belong to the same element $\omega_{j}^{-} \in M^{-}$, then conjugate $\gamma$ by $\gamma_{j}=f_{-}$. If the fixed points $f_{-} w_{1}, f_{-} w_{2}$ of a new element belong to the same element of $M^{-}$, repeat Step 2, if they belong to the same elernent of $M^{+}$, go to Step 1; otherwise proceed to Step 3. 
Step 3. Now the fixed points of $\gamma$ belong to different elements of both $\mathrm{M}^{+}$and $\mathrm{M}^{-}$. Therefore $C(\gamma)$ intersects the compact region $D_{0}$ and $\gamma$ is almost reduced. Since $D_{0} \subset \bigcup_{i=1}^{N} \gamma_{(i)} R_{0}, C(\gamma)$ intersects $\gamma(i) R_{0}$, for some $i$. Conjugate $\gamma$ by $\gamma_{(i)}^{-1}$ and obtain a reduced element.

\section{Remarks}

The algorithm described in Sect. 3 depends only on the geodesic $C(\gamma)$ and can be applied to any (not necessarily closed) geodesic in $D$. It allows us to reduce such a geodesic to obtain its code with respect to the given fundamental region $R_{0}$. The code will not be periodic unless the geodesic is closed, i.e., is an axis of a hyperbolic element in $\Gamma$.

Consider the arithmetic case where $\Gamma$ is contained in some quaternion algebra $H$ over $\mathbf{Q}$ with $H \otimes_{\mathbf{Q}} \mathbf{R}=M_{2}(\mathbf{R})$. Then to each $\alpha \in H$ which is hyperbolic [i.e., $(\operatorname{tr} \alpha)^{2}$ $-4 \operatorname{det} \alpha>0$ ] corresponds a geodesic $C(\alpha)$ whose image in $\Gamma \backslash D$ is closed since there exists a hyperbolic $\gamma \in \Gamma$ with $C(\gamma)=C(\alpha)$ (the centralizer of $\alpha$ in $H$ is a real quadratic field and $\gamma$ corresponds to a non-trivial unit). Note that any element $\lambda \alpha+\mu\left(\lambda \in \mathbf{Q}^{*}, \mu \in \mathbf{Q}\right)$ has the same geodesic. Consider the set of $\alpha \in H$ modulo the equivalence relation $\alpha \sim \lambda \alpha+\mu$. Choosing $\lambda$ suitably we can assume that $\alpha \in \mathcal{O}$, a given order of $H$, containing $\Gamma$, and that $\alpha$ is primitive (not divisible by an integer bigger than 1) in $\mathscr{2}=\mathcal{O} / \mathbf{Z} \simeq \mathbf{Z}^{3}$. The group $\Gamma$ acts on $\mathscr{Q}$ by conjugations. What our reduction algorithm does is to pick out of each $\Gamma$-equivalence class of hyperbolic elements of $\mathscr{Q}$ a canonical (finite and non-empty) set of representatives which form a cycle in a natural way. In the classical case $\Gamma=S L_{2}(\mathrm{Z}), H=M_{2}(\mathbf{Q}), \mathcal{O}=M_{2}(\mathrm{Z})$ the space 2 is the space of all binary quadratic forms with integer coefficients, and the analog of our theory is Gauss reduction theory of indefinite binary quadratic forms (as described, for instance, in [6, Chap. 13]).

\section{Examples}

1. The following example illustrates the algorithm of the construction of the fundamental region $R_{0}$ given in Sect. 1 for a special arithmetic group $\Gamma$. We begin from a subgroup of $P S L_{2}(\mathbf{R})$

$$
\Gamma_{15}=\left\{\gamma= \pm\left(\begin{array}{cc}
\frac{l+m \sqrt{3}}{2} & \sqrt{5}\left(\frac{w-u \sqrt{3}}{2}\right) \\
\sqrt{5}\left(\frac{w+u \sqrt{3}}{2}\right) & \frac{l-m \sqrt{3}}{2}
\end{array}\right),\right.
$$

where

$$
\left.\begin{array}{c}
(l, m, u, w) \in \mathbf{Z}^{4}, l \equiv w(\bmod 2), m \equiv u(\bmod 2) \text { and } \operatorname{det} \gamma=1, \\
\text { i.e. } l^{2}-3 m^{2}-5 w^{2}+15 u^{2}=4
\end{array}\right\}
$$


This group is an embedding of the group of units of a maximal order of the quaternion algebra over $Q$ with discriminant 15 [7, p. 123]. The group $\Gamma=R \Gamma_{15} R^{-1}$, where $R=\left(\begin{array}{ll}i & 1 \\ 1 & i\end{array}\right)$ acts on the unit disc $D$. Let us denote $R \gamma R^{-1}$ $=\left(\begin{array}{ll}a & \bar{c} \\ c & \bar{a}\end{array}\right)$. Then $a=\frac{l-i u \sqrt{15}}{2}, c=\frac{w \sqrt{5}-i m \sqrt{3}}{2},|a|^{2}=\frac{r}{4}+1, \quad|c|^{2}=\frac{r}{4}$, so $|a|^{2},|c|^{2} \in \frac{1}{4} \mathbf{Z}$. We can therefore list all elements $\left(\begin{array}{ll}a & \bar{c} \\ c & \bar{a}\end{array}\right)$ of $\Gamma$ in increasing order of $|a|$ by solving the equations $l^{2}+15 u^{2}=r+4,5 w^{2}+3 m^{2}=r, l \equiv w(\bmod 2)$, $m \equiv u(\bmod 2)$ for $r=0,1,2 \ldots$.

Table 1

\begin{tabular}{|c|c|c|c|c|c|c|c|}
\hline$x$ & $\ell$ & m & $u$ & $w$ & $x$ & $y$ & $\mathbf{R}$ \\
\hline $\begin{array}{l}5 \\
5 \\
12 \\
12 \\
27 \\
27 \\
27 \\
27 \\
32 \\
32 \\
32 \\
32 \\
45 \\
45 \\
47 \\
47 \\
47 \\
47 \\
47 \\
47 \\
47 \\
47 \\
57 \\
57 \\
57 \\
57 \\
57 \\
57 \\
57 \\
57 \\
75 \\
75 \\
75 \\
75 \\
92 \\
92 \\
92 \\
92 \\
92 \\
92 \\
92 \\
92 \\
137 \\
137 \\
137 \\
137 \\
137 \\
137 \\
137 \\
137\end{array}$ & $\begin{array}{l}3 \\
3 \\
4 \\
4 \\
4 \\
4 \\
4 \\
4 \\
6 \\
6 \\
6 \\
6 \\
7 \\
7 \\
6 \\
6 \\
6 \\
6 \\
6 \\
6 \\
6 \\
6 \\
1 \\
1 \\
1 \\
1 \\
1 \\
1 \\
1 \\
1 \\
8 \\
8 \\
8 \\
8 \\
6 \\
6 \\
6 \\
6 \\
6 \\
6 \\
6 \\
6 \\
9 \\
9 \\
9 \\
9 \\
9 \\
9 \\
9 \\
9\end{array}$ & $\begin{array}{r}0 \\
0 \\
-2 \\
2 \\
-3 \\
3 \\
-3 \\
3 \\
-2 \\
-2 \\
2 \\
2 \\
0 \\
0 \\
-3 \\
-3 \\
3 \\
3 \\
-3 \\
-3 \\
3 \\
3 \\
-2 \\
-2 \\
2 \\
2 \\
-2 \\
-2 \\
2 \\
2 \\
-5 \\
5 \\
-5 \\
5 \\
-2 \\
-2 \\
2 \\
2 \\
-2 \\
-2 \\
2 \\
2 \\
-2 \\
-2 \\
2 \\
2 \\
-2 \\
-2 \\
2 \\
2 \\
\end{array}$ & $\begin{array}{r}0 \\
0 \\
0 \\
0 \\
-1 \\
-1 \\
1 \\
1 \\
0 \\
0 \\
0 \\
0 \\
0 \\
0 \\
-1 \\
-1 \\
-1 \\
-1 \\
1 \\
1 \\
1 \\
1 \\
-2 \\
-2 \\
-2 \\
-2 \\
2 \\
2 \\
2 \\
2 \\
-1 \\
-1 \\
1 \\
1 \\
-2 \\
-2 \\
-2 \\
-2 \\
2 \\
2 \\
2 \\
2 \\
-2 \\
-2 \\
-2 \\
-2 \\
2 \\
2 \\
2 \\
2 \\
\end{array}$ & $\begin{array}{r}-1 \\
1 \\
0 \\
0 \\
0 \\
0 \\
0 \\
0 \\
-2 \\
2 \\
-2 \\
2 \\
-3 \\
3 \\
-2 \\
2 \\
-2 \\
2 \\
-2 \\
2 \\
-2 \\
2 \\
-3 \\
3 \\
-3 \\
3 \\
-3 \\
3 \\
-3 \\
3 \\
0 \\
0 \\
0 \\
0 \\
-4 \\
4 \\
-4 \\
4 \\
-4 \\
4 \\
-4 \\
4 \\
-5 \\
5 \\
-5 \\
5 \\
-5 \\
5 \\
-5 \\
5 \\
\end{array}$ & $\begin{array}{r}1.342 \\
-1.342 \\
0.000 \\
0.000 \\
0.745 \\
-0.745 \\
-0.745 \\
0.745 \\
0.839 \\
-0.839 \\
0.839 \\
-0.839 \\
1.043 \\
-1.043 \\
0.999 \\
-0.143 \\
0.143 \\
-0.999 \\
0.143 \\
-0.999 \\
0.999 \\
-0.143 \\
0.588 \\
0.353 \\
-0.353 \\
-0.588 \\
-0.353 \\
-0.588 \\
0.588 \\
0.353 \\
0.447 \\
-0.447 \\
-0.447 \\
0.447 \\
0.875 \\
-0.292 \\
0.292 \\
-0.875 \\
0.292 \\
-0.875 \\
0.875 \\
-0.292 \\
0.930 \\
-0.539 \\
0.539 \\
-0.930 \\
0.539 \\
-0.930 \\
0.930 \\
-0.539 \\
\end{array}$ & $\begin{array}{r}0.000 \\
0.000 \\
1.155 \\
-1.155 \\
0.770 \\
-0.770 \\
0.770 \\
-0.770 \\
0.650 \\
0.650 \\
-0.650 \\
-0.650 \\
0.000 \\
0.000 \\
0.295 \\
1.032 \\
-1.032 \\
-0.295 \\
1.032 \\
0.295 \\
-0.295 \\
-1.032 \\
-0.851 \\
0.972 \\
-0.972 \\
0.851 \\
0.972 \\
-0.851 \\
0.851 \\
-0.972 \\
0.924 \\
-0.924 \\
0.924 \\
-0.924 \\
-0.527 \\
0.979 \\
-0.979 \\
0.527 \\
0.979 \\
-0.527 \\
0.527 \\
-0.979 \\
-0.405 \\
0.860 \\
-0.860 \\
0.405 \\
0.860 \\
-0.405 \\
0.405 \\
-0.860 \\
\end{array}$ & $\begin{array}{l}0.894 \\
0.894 \\
0.577 \\
0.577 \\
0.385 \\
0.385 \\
0.385 \\
0.385 \\
0.354 \\
0.354 \\
0.354 \\
0.354 \\
0.298 \\
0.298 \\
0.292 \\
0.292 \\
0.292 \\
0.292 \\
0.292 \\
0.292 \\
0.292 \\
0.292 \\
0.265 \\
0.265 \\
0.265 \\
0.265 \\
0.265 \\
0.265 \\
0.265 \\
0.265 \\
0.231 \\
0.231 \\
0.231 \\
0.231 \\
0.209 \\
0.209 \\
0.209 \\
0.209 \\
0.209 \\
0.209 \\
0.209 \\
0.209 \\
0.171 \\
0.171 \\
0.171 \\
0.171 \\
0.171 \\
0.171 \\
0.171 \\
0.171 \\
\end{array}$ \\
\hline
\end{tabular}

In Table 1 we give the beginning of this list. (For $r=0$ we get the identity element which we do not include in the table.) Columns 1-5 give values of $r, l, m, u, w$. Columns 6-8 give the coordinates $(x, y)$ of the center of the corresponding isometric circle and its radius $R$. The isometric circles of the first 8 elements form a boundary of the fundamental region $R_{0}$ (see Fig. 3), and therefore those elements can be chosen as generators of the group $\Gamma$. The genus of $\Gamma \backslash D$ is 1 and the number of non-equivalent in $\Gamma$ elliptic points of order 3 equals 2 . The special 
Fig. 3
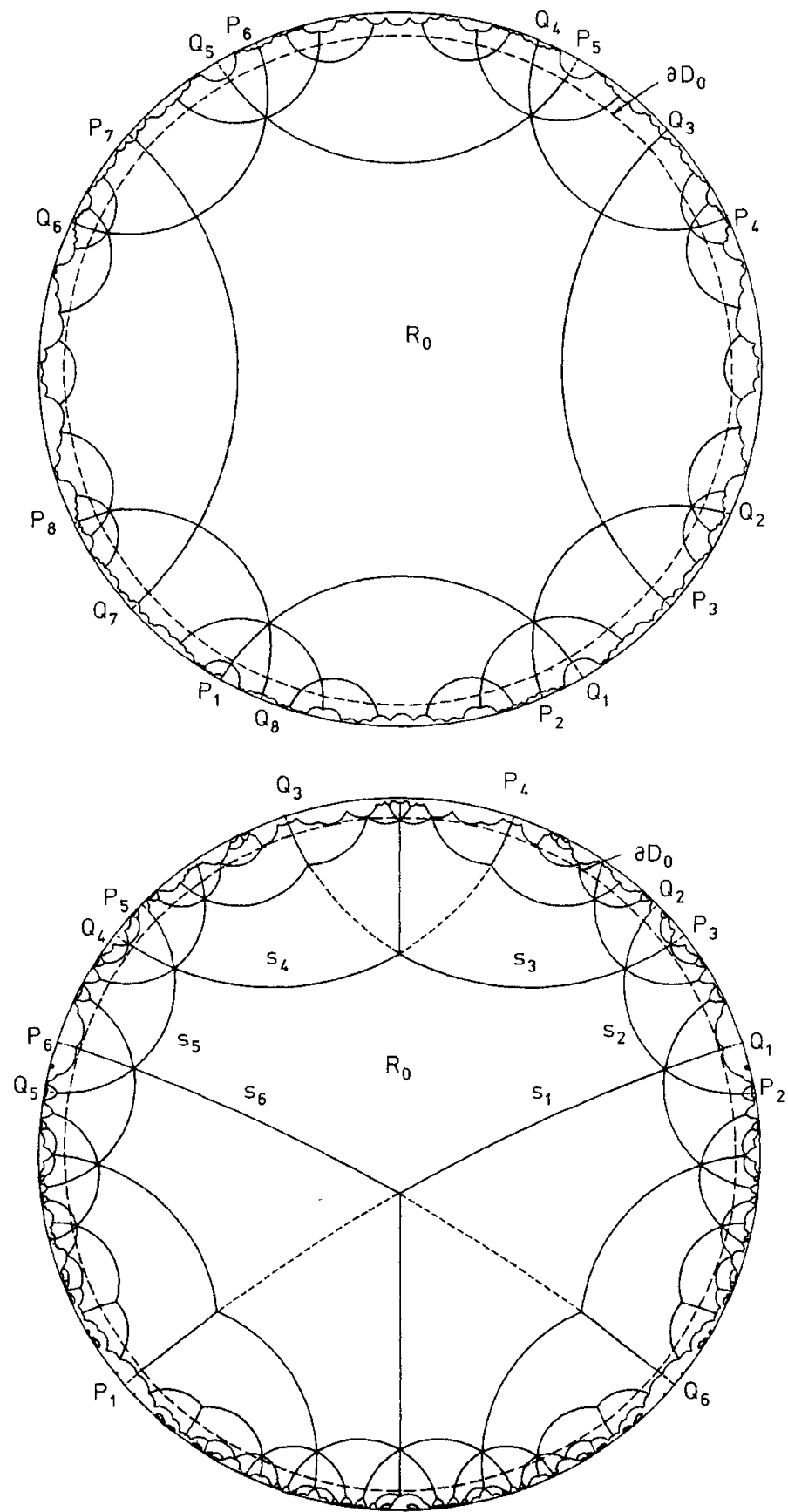

polygon $R$ in this example coincides with the fundamental region $R_{0}$. The compact region $D_{0}$ described in Sect. 3 and a finite part of the tesselation of $D$ by the images of $R_{0}$ is given in Fig. 3 .

2. In Fig. 4 we give the fundamental region $R_{0}$, the tesselation of $D$ and the compact region $D_{0}$ for $\Gamma=R \Gamma_{10} R^{-1}$, where $\Gamma_{10}$ is the following arithmetic 
Table 2

\begin{tabular}{|c|c|c|c|c|c|c|c|}
\hline Side of $R_{0}$ & $\ell$ & $m$ & $u$ & & $\mathrm{x}$ & $\mathrm{y}$ & $\mathrm{R}$ \\
\hline$s_{1}$ & 1 & 0 & -1 & 1 & 2.581 & -4.581 & 5.162 \\
\hline$s_{2}$ & 6 & 0 & 4 & -16 & 0.930 & 0.481 & 0.310 \\
\hline$s_{3}$ & 1 & 0 & 3 & -9 & 0.352 & 1.172 & 0.705 \\
\hline$s_{4}$ & 1 & 0 & -3 & 9 & -0.352 & 1.172 & 0.705 \\
\hline$s_{5}$ & 6 & 0 & -4 & 16 & -0.930 & 0.481 & 0.310 \\
\hline$s_{6}$ & 1 & 0 & 1 & -1 & -2.581 & -4.581 & 5.162 \\
\hline
\end{tabular}

Table 3

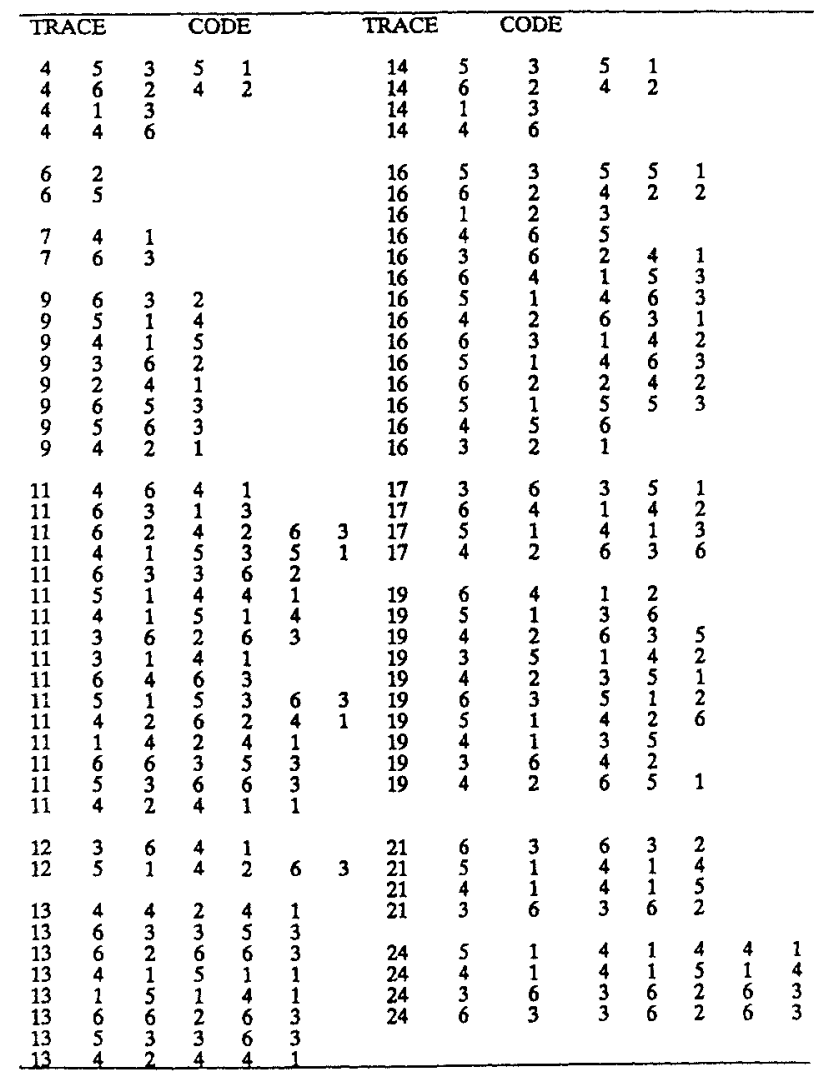


subgroup of $P S L_{2}(\mathbf{R})$ :

$$
\Gamma_{10}=\left\{\gamma= \pm\left(\begin{array}{cc}
\frac{l+m \sqrt{10}}{2} & \frac{w-u \sqrt{10}}{6} \\
\frac{w+u \sqrt{10}}{2} & \frac{l-m \sqrt{10}}{2}
\end{array}\right),\right.
$$

where

$$
\left.\begin{array}{c}
(l, m, u, w) \in Z^{4}, 3 \mid(u+w), l-n \equiv \frac{u+w}{3} \equiv m(\bmod 2), \text { and } \operatorname{det} \gamma=1, \\
\text { i.e. } 3 l^{2}-30 m^{2}+10 u^{2}-w^{2}=12
\end{array}\right\} .
$$

The set of generators of $\Gamma$ whose isometric circles form the boundary of the fundamental region $R_{0}$ is given in Table 2 .

Table 3 gives codes of all elements of $\Gamma$ with traces up to 24 with respect to the fundamental region $R_{0}$, as explained at the beginning of Sect. 3. This group has been studied in great detail in [3, Chap. 4]. Here we give several examples of elements of trace 4. Elements $\gamma_{1}=(4,0,8,-26)$ and $\gamma_{2}=(4,1,43,-136)$ have different codes: code $\gamma_{1}=(13)$, $\operatorname{code} \gamma_{2}=(6242)$ and therefore they are not conjugate in $\Gamma$ and represent different closed geodesics. Elements $\gamma_{3}=(4,1,1,-4)$ and $\gamma_{4}=(4,0,-8,26)$ have the same code $(46)$ and therefore are conjugate in $\Gamma$. For trace 4 there are four different closed geodesics, (5351) and (6242), (13) and (46), the geodesics in each pair differ only in orientation.

Acknowledgements. I am indebted to Don Zagier who brought my attention to this problem and encouraged me to work on it by his insight. I also would like to thank Caroline Series for stimulating discussions on Markov maps. The computer calculations reported in Sect. 5 were made during my visit in the University of Warwick in July 1984. I would like to thank Ben Mestel for his help with these calculations.

\section{References}

1. Bowen, R., Series, C.: Markov maps associated with Fuchsian groups. Publ. Math. Inst. Hautes Étud. Sci. 50, 401-418 (1978)

2. Ford, L.: Automorphic functions. New York: Chelsea 1951

3. Katok, S.: Modular forms associated to closed geodesics and arithmetic applications. Ph. D. Thesis, University of Maryland (1983)

4. Morse, M.: Symbolic dynamics. Institute for Advanced Study Notes, Princeton (1966) (unpublished)

5. Series, C.: Symbolic dynamics for geodesic flows. Acta Math. 146, 103-128 (1981)

6. Zagier, D.: Zetafunktionen und quadratische Körper: eine Einführung in die höhere Zahlentheorie. Hochschultext, Berlin, Heidelberg, New York: Springer 1982

7. Vignéras, M.F.: Arithmétic des algèbres de quaternions. Lect. Notes Math. 800. Berlin, Heidelberg, New York: Springer 1980 